\title{
Embracing Change: The Evolving Science of Relative Energy Deficiency in Sport
}

\author{
Megan A. Kuikman \\ University of Guelph \\ Margo Mountjoy \\ McMaster University \\ Trent Stellingwerff \\ Jamie F. Burr \\ Canadian Sport Institute Pacific and University of Victoria University of Guelph
}

Firstly, we would like to thank Dr. Strock, Koltun, and Ricker for their interest in our article (Kuikman et al., 2021) and for expressing their opinion on our review of treatment approaches to Relative Energy Deficiency in Sport (RED-S). We also appreciate the opportunity to address their concerns and expand on the global direction of research in this area. Building upon the foundational research on the Female Athlete Triad (TRIAD), scientific knowledge of RED-S is rapidly expanding and evolving as the understanding of contributing and interacting factors increases. As the evidence base grows, researchers and members of the athlete support team must adapt their theories and frameworks.

While this review was intended to highlight the state-of-the-art treatment approaches currently available, Strock et al. (2021) instead deemed this paper inaccurate and misleading for encapsulating the terminology of the TRIAD within the RED-S model. It was in no way the intention of the Authors of this review to mislead nor neglect important work; instead, the progress and global acceptance of RED-S was written with the assumption that the TRIAD model was captured within the definition of RED-S as the TRIAD had been previously included in both consensus statements (Mountjoy et al., 2014, 2018). We find the suggestion that low energy availability (EA), menstrual dysfunction, and bone health can only be discussed within the context of the TRIAD to be misleading and somewhat nonsensical as these topics have been included within the RED-S model since its inception (Mountjoy et al., 2014, 2015, 2018). Further, as an increasing number of athletes come forth to share their struggles with RED-S (Ackerman et al., 2020), the use of fragmented definitions or scientific ideology on this topic could cause a significant disservice to athletes that may require future assistance.

Concerns were also raised by the reviewers relating to "omitting" select papers (Strock et al., 2021). The authors attest that this review was, in fact, very comprehensive as it included papers from the TRIAD literature as well as several recent articles that have changed our understanding of this complex issue. Some of the

Kuikman and Burr are with The Human Performance and Health Research Laboratory, Department of Human Health and Nutritional Sciences, University of Guelph, Guelph, Ontario, Canada. Mountjoy is with the Department of Family Medicine, Michael G. DeGroote School of Medicine, McMaster University, Hamilton, Ontario, Canada. Stellingwerff is with the Canadian Sport Institute Pacific, Victoria, British Columbia, Canada; and the Department of Exercise Science, Physical \& Health Education, University of Victoria, British Columbia, Canada. Burr is also with Animal Science and Nutrition, University of Guelph, Guelph, Ontario, Canada. Burr (burrj@uoguelph.ca) is corresponding author. specific articles noted as being "omitted" were not considered relevant to the purpose of this review, as they either interfered with word count limitations, or their topics had already been discussed extensively elsewhere. For instance, including studies that assessed the EA thresholds was considered unnecessary because achieving this threshold was not considered the goal of treatment. As outlined, improving EA is necessary for the treatment of RED-S as this is the proposed underlying cause (Kuikman et al., 2021). However, athletes do not live in a laboratory, and they need real-world strategies. The highlighted nutritional interventions reflected practical strategies that, in many cases, targeted the underlying causes of low EA, and as such, would simultaneously improve it. Treating RED-S, and failing to address other areas that may be co-contributing to the deleterious health outcomes, such as inadequate intake of bone-building micronutrients, could be neglecting the best interest of the athletes.

To suggest that there is insufficient research to support the RED-S model excludes extensive previous research that has demonstrated multiple deleterious outcomes outside of the TRIAD, including studies involving male subjects. Moreover, dismissing the RED-S model potentially keeps athletes that fall outside of the TRIAD model from being able to receive help. This neglects the real-life situations of other athlete groups beyond naturally menstruating females, such as post-menopausal females or males with hormonal imbalances, who can also suffer from the consequences of low EA. It is our strong belief that athletes are deserving of evidence-based, best-practice treatment irrespective of the label given to their diagnosis. We need to move on and accept the evolving science. To this point, we appreciate the perspective of Dr. Barbara Drinkwater, whose seminal research set the stage for the TRIAD, who stated:

I think RED-S is the natural evolution as more information and studies have come about. We need to move on. Any athlete with energy deficiency needs to be identified and be given help, above all else. The more cohesive the sports medicine community is, the better it will be for the athletes. Collegiality will accelerate our cause - the more working towards the same goal, the better. In unison we can speed up delivering the treatment that these athletes so desperately need (Carter, 2018).

\section{References}

Ackerman, K.E., Stellingwerff, T., Elliott, K.J., Baltzell, A., Cain, M., Goucher, K., \& Mountjoy, M.L. (2020). \#REDS (Relative Energy 
Deficiency in Sport): Time for a revolution in sports culture and systems to improve athlete health and performance. British Journal of Sports Medicine, 54(7), 1-2. PubMed ID: 31924625 doi:10.1136/ bjsports-2019-101926

Carter, S. (2018). Female athlete triad/relative energy deficiency in sport: A perspective interview with Professor Barbara Drinkwater. International Journal of Sport Nutrition and Exercise Metabolism, 28(4), 332-334. PubMed ID: 29485333 doi:10.1123/ijsnem.20180030

Kuikman, M.A., Mountjoy, M., Stellingwerff, T., \& Burr, J.F. (2021). A review of nonpharmacological strategies in the treatment of relative energy deficiency in sport. International Journal of Sport Nutrition and Exercise Metabolism, 31(3), 268-275. doi:10.1123/ijsnem. 2020-0211

Mountjoy, M., Sundgot-Borgen, J., Burke, L., Carter, S., Constantini, N., Lebrun, C., .. L Ljungqvist, A. (2014). The IOC consensus statement: Beyond the female athlete triad-relative energy deficiency in sport
(RED-S). British Journal of Sports Medicine, 48(7), 491-497. PubMed ID: 24620037 doi:10.1136/bjsports-2014-093502

Mountjoy, M., Sundgot-Borgen, J., Burke, L., Carter, S., Constantini, N., Lebrun, C., ... Ljungqvist, A. (2015). Authors' 2015 additions to the IOC consensus statement: Relative Energy Deficiency in Sport (REDS). British Journal of Sports Medicine, 49(7), 417-420. PubMed ID: 25950026 doi:10.1136/bjsports-2014-094371

Mountjoy, M., Sundgot-Borgen, J.K., Burke, L.M., Ackerman, K.E., Blauwet, C., Constantini, N., Budgett, R. (2018). IOC consensus statement on relative energy deficiency in sport (RED-S): 2018 update. British Journal of Sports Medicine, 52(11), 687-697. PubMed ID: 29773536 doi:10.1136/bjsports-2018-099193

Strock, N.C.A., Koltun, K.J., \& Ricker, E.A. (2021). Commentary in response to "a review of nonpharmacological strategies in the treatment of relative energy deficiency in sport." International Journal of Sport Nutrition \& Exercise Metabolism, 31(4). doi:10.1123/ijsnem. 2021-0084 